\title{
Isolation and Identification of Candida Species from Various Clinical Samples and their Biofilm Production Status
}

\author{
S. Prateeksha ${ }^{1}$, D.C. Shwetha ${ }^{2 *}$ and D. Venkatesha ${ }^{2}$ \\ ${ }^{1}$ Adichunchanagiri Institute of Medical Sciences, AIMS, BG nagara, Mandya -571448, India \\ ${ }^{2}$ Department of Microbiology, AIMS, B G Nagara, Mandya-571448, India \\ *Corresponding author
}

Ke y w o r d s
Candida species,
Clinical samples,
Biofilm production
status

\section{Introduction}

Fungal infections are considered a serious health problem and are a major cause of morbidity and mortality worldwide. Among all the fungal pathogens, Candida species are a major cause of morbidity and mortality worldwide and thus represents a serious threat to public health (De Oliveira Santos et al., 2018).

\section{A B S T R A C T}

Candida species are the most common fungal species causing mucosal and systemic infections. Candida albicans has been the most common causative agent affecting mostly the immunocompromised. However, an increase in the prevalence of Non Albicans Candida (NAC) species is noted in the recent two decades. In this study Candida species from various clinical samples were isolated and their biofilm production status was evaluated. All the clinical samples were inoculated on to Blood agar and MacConkey agar. The suspected colonies of Candida were further confirmed by Gram stain. Germ tube test was done for the differentiation of $C$. albicans and $C$. dublinensis from the other candida species. It was further inoculated onto HiCrome Candida Differential agar for the speciation. The Biofilm production was done using the Congo-red agar. Out of a total 36 candida species that were isolated during the study, 18 (50\%) were found to be Candida albicans and remaining 18 were found to be NAC (22.2\% were $C$. tropicalis, $19.4 \%$ were $C$. glabrata, $8.4 \%$ were $C$. krusei). Among the 36 candida species, 12 (33\%) were biofilm producers while the remaining $24(64 \%)$ were non-biofilm producers. This study showed that there is an increase in the pathogenicity and prevalence of the NAC species. 
oropharynx, female genital tract and skin (Sandhu et al., 2015). Candida species are responsible for various clinical manifestations ranging from mucocutaneous overgrowth to life threatening disseminated infections like candidemia (Deorukhkar et al., 2014). The pathogenicity of Candida species is attributed to certain virulence factors, such as the ability to evade host defenses, adherence, biofilm formation (on host tissue and on medical devices) and the production of tissue damaging hydrolytic enzymes such as proteases, phospholipases and hemolysin (Sardi et al., 2013).

While Candida albicans is still the most common causative agent of nosocomial fungal infections, some studies have reported increasing rates of species other than $C$. albicans (Aydemir et al., 2017). Shifting of trend to Non albicans Candida (NAC) infection with high antifungal resistance has been witnessed (Chandak et al., 2018). The Non albicans Candida species includes $C$. tropicalis, C. glabrata, C. parapsilosis, $C$. krusei and others. Several brands of chromogenic media have been developed to produce rapid yeast identification. These media contain chromogenic substrates that react with the enzymes secreted by microorganisms producing colonies showing various pigmentations. These enzymes are species specific, allowing organisms to be identified to the species level by their colour and colony characteristics (Sandhu et al., 2015).

Biofilm aids the producing organism to withstand or evade host defense mechanism and enables the organism to survive and exist as reservoir and recurrent source of infection as well as development of resistance to antimicrobial agents (Das et al., 2016). The ability of Candida species to form drugresistant biofilms is an important factor in their contribution to human disease. Decision to treat such patients with antifungal drugs is complex (Naveen Saxena et al., 2014). There are various methods used to detect biofilm production like Congo red agar method, determination of dry weight, scanning electron microscope (SEM) etc. (Dhale et al., 2014).

In the recent years, the incidence of nosocomial candidiasis has increased throughout the world, starting from tertiary care centers and spreading to community hospitals (Kaur et al., 2016). The clinical manifestations of infections caused by different members of NAC species are usually indistinguishable and several NAC species are inherently resistant or acquire resistance or both, to commonly used antifungal drugs (Deorukhkar et al., 2014). This variability increases the challenge of finding an effective solution to tackle the threats of Candida biofilms as a unique problem (Cavalheiro et al., 2018). Hence, identification of Candida species and detection of biofilms becomes necessary.

Thus, this study aim to isolate and identify Candida species from various clinical samples and also to detect the biofilm production of isolated Candida species.

\section{Materials and Methods}

This study was conducted over a period of 2 months (May and June) in the department of Microbiology, Adichunchangiri Institute of Medical Sciences, Mandya. A total of 36 isolates were recovered from clinical samples like High vaginal swab(HVS), sputum samples, urine samples and Endotracheal tubes. Patients on any form of antifungal therapy 6 weeks prior to sample collection were excluded from the study.

All the clinical samples were inoculated onto blood agar and MacConkey agar and 
incubated aerobically at $37^{\circ} \mathrm{C}$ for $24-48$ hours. Colonies appearing pasty, opaque, slightly domed, white or off-white coloured is suspected as the colonies of Candida and is identified by Gram stain. The one which revealed Gram positive budding yeast cells was inoculated on SDA (Sabouraud Dextrose agar) and HiCrome Candida differential agar procured from Himedia Pvt. Ltd, Mumbai, India.

\section{Inoculation on Sabouraud Dextrose agar (SDA)}

The growth obtained on SDA culture medium was identified based on colony morphology, Gram stain and subjected to germ tube test.

\section{Rapid germ tube test}

This test was done to differentiate $C$. albicans and $C$. dublinenses from other Candida species. Small inoculums of yeast cells obtained from an isolated colony were suspended in $0.5 \mathrm{ml}$ of serum \& was incubated at $37^{\circ} \mathrm{C}$ for no longer than 3 hours. A drop of this suspension was placed on a microscope slide \& examined for the presence of germ tubes (Forbes et al., 2007).

\section{Inoculation on HiCrome Candida differential agar}

Further speciation of the isolates was done by culturing it on HiCrome Candida differential agar. HiCrome agar was prepared as per the manufacturer's instructions and incubated at $30^{\circ} \mathrm{C}$ for $24-48$ hours. Species identification was done by the morphology and colour of the colony (chromogenic reaction). The isolates that remained doubtful in their appearance on HiCrome agar was considered as unidentified and excluded from the study.

The repeated isolation of Candida species from clinical specimen like oropharyngeal, vaginal, urinary and bronchial candidiasis was considered significant. While a single isolation was considered significant from sterile body fluids like blood, peritoneal fluid, pleural fluid and CSF.

The in-vitro screening test for biofilm production in Candida isolates was carried out using Congo red agar method. The Composition of the Congo red agar media is BHI (37 gm/L), glucose (80 gm/L), agar no.1 $(10 \mathrm{gm} / \mathrm{L})$ and Congo red stain $(0.8 \mathrm{gm} / \mathrm{L})$. Aqueous solution of Congo red was prepared and autoclaved separately and added to the agar after cooling it to $55^{\circ} \mathrm{C}$. The identified Candida species were inoculated on plates of Congo red agar and incubated aerobically at $37^{\circ} \mathrm{C}$ for 24-48 hours. Positive biofilm production by Candida species was indicated by the appearance of black colonies, whereas biofilm negative Candida species produces white or very light pink colonies.

\section{Results and Discussion}

In the present study 36 Candida species were isolated over a period of two months (May and June 2019) from various clinical samples such as High vaginal swab (HVS), urine, sputum samples and Endotracheal tube as shown in Table 1. All the isolates were subjected to speciation and biofilm formation. HiCrome agar showed good growth of all the Candida isolates after 48 hours of incubation.

Candida species are part of normal human flora and are opportunists capable of causing a wide spectrum of infections. Colonisation of the mucocutaneous surfaces is the first step towards infection. Alteration in this balance results in growth and subsequent invasion and is supported by various risk factors leading to immunosuppression. Some of these include infections with HIV/AIDS, indiscriminate antibiotic use, use of intravenous catheters, urinary tract catheterisation, hepatic and renal 
failure, prolonged hospitalstay, chemotherapy, organ transplant, leukaemia, diabetes mellitus and Chronic Obstructive Pulmonary Diseases (COPD).

Candida albicans has been the most common species causing infection for many years but, indiscriminate use of azole group of drugs has led to increase in NAC infection. Hence, infections with NAC and overall resistance to antifungals are on the rise. This makes species identification of Candida very essential to prevent treatment failures (Jangla et al., 2018).

Conventional Candida speciation methods like morphology on Corn meal agar, carbohydrate fermentation and assimilation tests are time consuming taking from 72 hours to 2 weeks and the procedures are labor intensive (Kaup et al., 2016). The longer turnaround time taken by conventional methods of identification makes them less popular among the clinicians as early diagnosis is essential for initiating appropriate therapy (Shettar et al., 2012). The need for rapid identification of Candida species and the difficulty in detecting mixed cultures of Candida on Sabouraud's Dextrose Agar, has led to the development of several methods that differentiate yeast species. Newer methods which have been developed for yeast identification include CHROM agar, API systems, Vitek 2 ID system and molecular methods. Several brands of chromogenic media have been developed to produce rapid yeast identification. These media contain chromogenic substrates that react with enzymes secreted by microorganisms producing colonies with various pigmentation. These enzymes are species specific, allowing organisms to be identified to the species level by their colour and colony characteristics (Horvath et al., 2003).

HiCrome Candida differential agar is one such medium introduced by Himedia laboratory to differentiate Candida species namely $C$. albicans, $C$. krusei, C. tropicalis, and $C$. glabrata based on colony colour. The colony color produced by Candida albicans, Candida tropicalis, Candida krusei, and Candida glabrata were typical. Candida albicans produces light green colonies, Candida tropicalisblue to metallic blue colonies, Candida krusei produces purple fuzzy colonies and Candida glabrata white to cream colored colonies (Figure 1). This medium can be recommended for identification of these species in resource limited settings as it will not require any expertise (Rachana Mehta et al., 2016). Hence, the present study was undertaken to identify Candida isolated from various clinical specimens to the species level using HiCrome Candida differential agar along with their biofilm production status.

In the present study, out of the 36 Candida species which were isolated from the specimen, 18 (50\%) were found to be Candida albicans and remaining 18 (50\%) were identified as Non albicans Candida. Among the $18(50 \%)$ NAC species, eight (22.2\%) were identified as $C$. tropicalis, seven $(19.4 \%)$ as $C$. glabrata and three $(8.4 \%)$ as $C$. krusei (Figure 2). Similar findings were reported by Sharma et al., where out of 120 Candida species, 58(48.33\%) were Candida albicans and 62 $(51.66 \%)$ were identified as NAC. Das et al., (2016) found similar distribution of Candida albicans 33(36.7\%) and NAC 57(63.3\%) from various clinical samples thus concluding that there is a notable shift in the pathogenic incidence of species from C.albicans to NAC species.C. albicans is the most frequently isolated species. Among the NAC species, $C$. tropicalis is most frequently isolated. This preponderance of $C$. tropicalis is consistent with other studies (Vignesh Kanna et al., 2017; Deorukhkar et al., 2014. Das et al., 2016). 
HiCrome agar has several advantages like rapidity, direct identification of species; thus, making it very useful in early identification and thereby early initiation of appropriate antifungal therapy (Shettar et al., 2012). A major advantage of chrome agar is the ability to detect mixed cultures of yeasts in clinical specimens (Manjunath et al., 2012). However, present study did not show any mixed isolates. In our study, Candida species were isolated from various clinical samples like HVS, urine, sputum. To rule out colonisation, a repeat sample was collected and processed to confirm the etiological significance of the isolate.
Figure 3 shows the distribution of Candida species from various clinical samples. In sputum samples, two were C. albicans and one was NAC. From HVS samples, eleven were $C$. albicans and five were NAC. From urine samples, five were $C$. albicans and eleven were NAC. One NAC was isolated from endotracheal aspiration fluid. Most of the Candida albicans species were found to be isolated from HVS samples while most of the Non albicans Candida (NAC) species were isolated from urine.

Table.1 Candida species isolated from various clinical samples

\begin{tabular}{|l|l|}
\hline Sample Type & Number of samples \\
\hline High vaginal swabs & 16 \\
\hline Urine & 16 \\
\hline Sputum & 03 \\
\hline ET Tube aspiration & 01 \\
\hline Total & $\mathbf{3 6}$ \\
\hline
\end{tabular}

Figure.1 Hicrome agar showing colonies of various candida species producing different colors

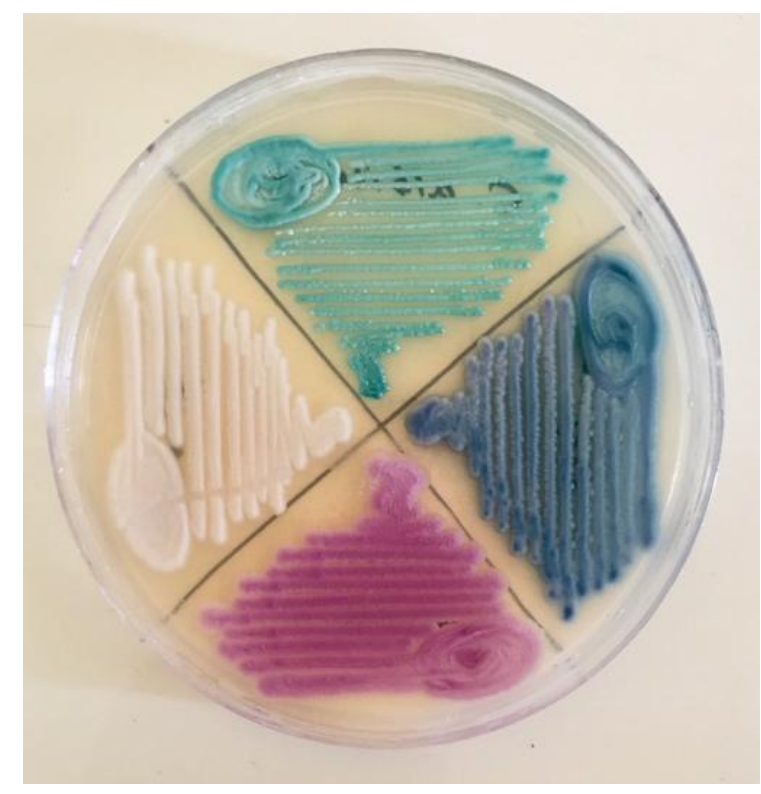


Figure.2 Distribution of Candida species

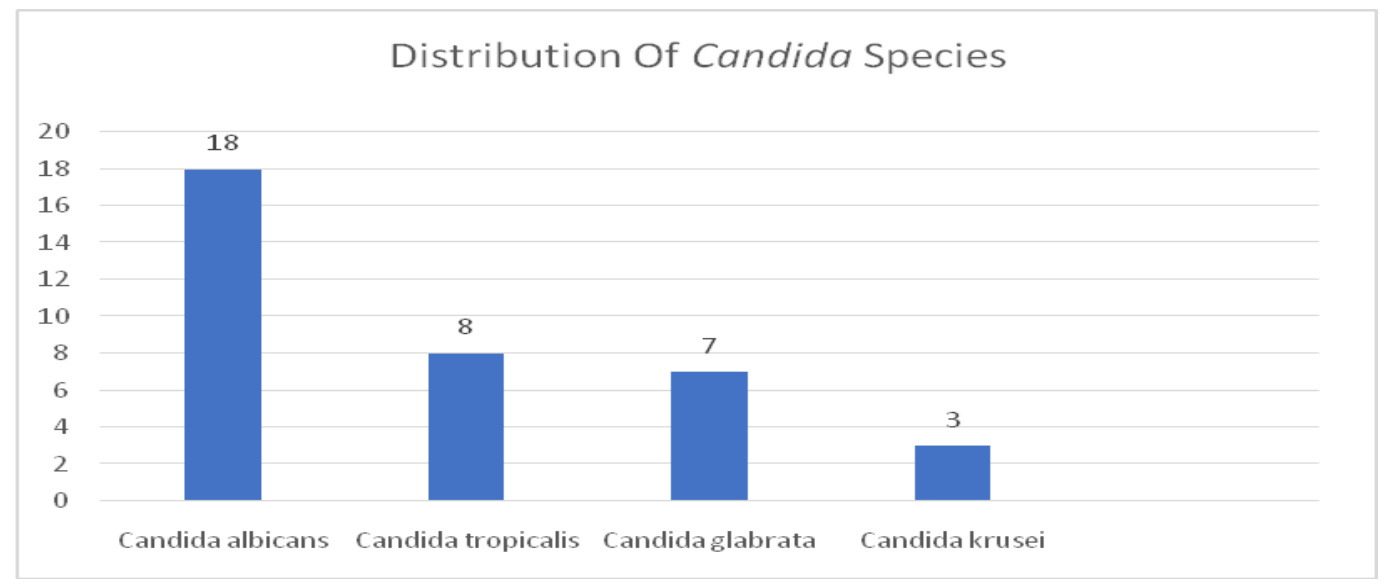

Figure.3 Distribution of Candida species in different clinical samples

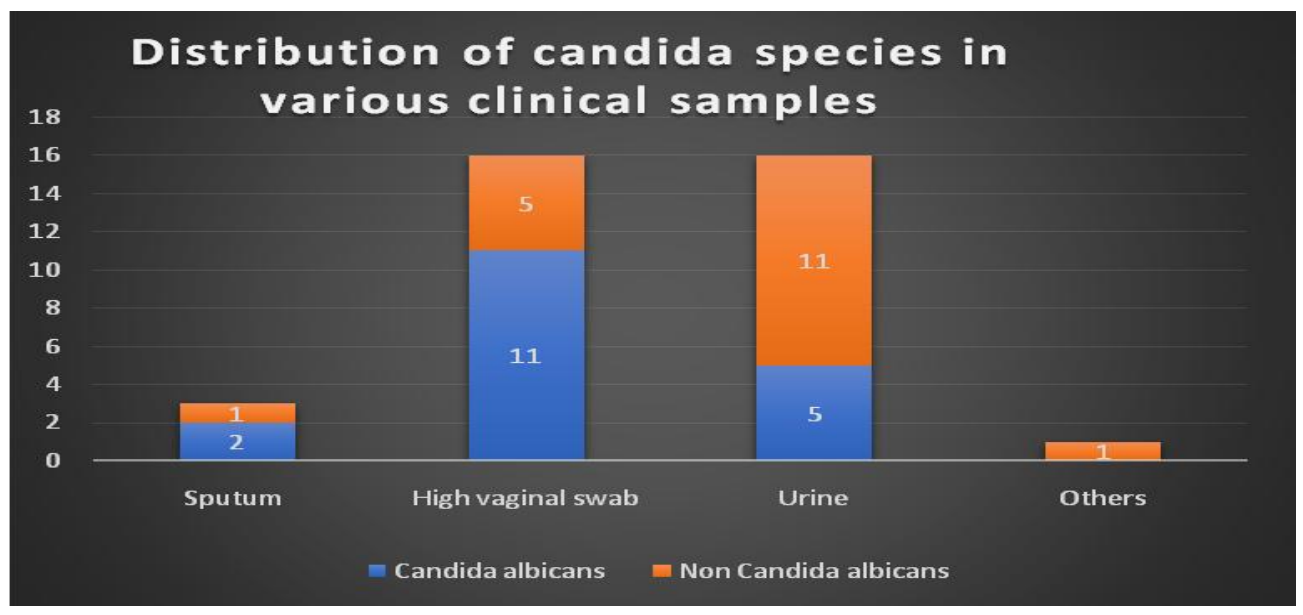

Figure.4 Biofilm producing Candida species

\section{DISTRIBUTION OF BIOFILM PRODUCERS}

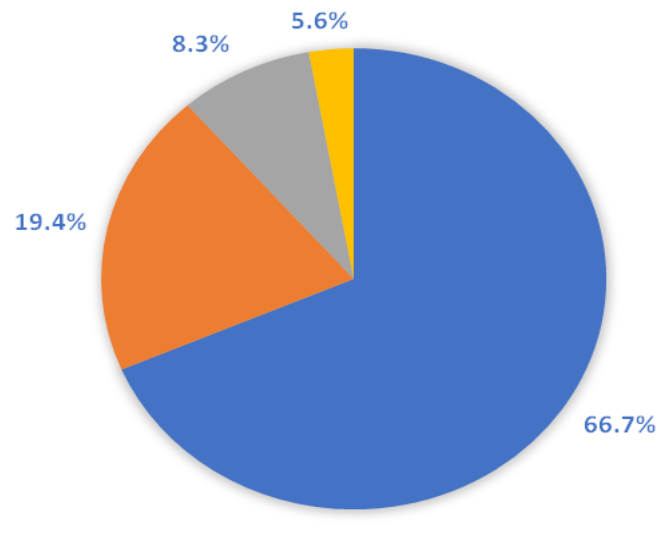


Figure.5 Congo red agar showing biofilm producing candida species and non-biofilm producing candida species

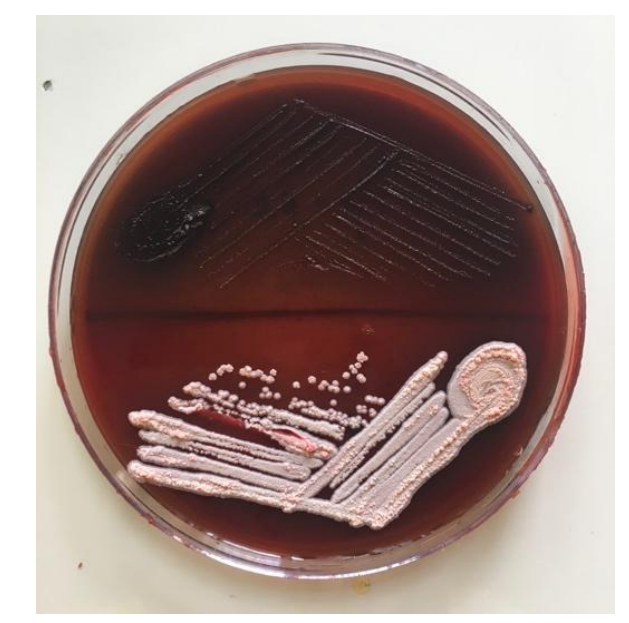

All the isolated Candida species were studied for biofilm production on Congo red agar. Among the 36 Candida species, 12 (33.3\%) were biofilm producers while the remaining $24(66.7 \%)$ were non biofilm producers. The biofilm producing species includes $7(19.4 \%)$ C. albicans, $3(8.3 \%)$ C. glabrata and 2 (5.6\%) C. tropicalis as depicted in Figure 4. This correlates with the study done by Saxena et al., where $38.3 \%$ of the isolates were biofilm producers. However slightly higher percentage is observed in other studies done by Sharma et al., (2017) and Marak et al., (2018) (52.5\% and $54.4 \%$ respectively). The figure 5 shows biofilm positive candida species with black colonies and biofilm negative candida species with pink colored colonies on Congo red agar.

Biofilms are universal, complex, interdependent communities of surfaceassociated microorganisms, enclosed in an exopolysaccharide matrix occurring on any surface, including medical devices. The pathogenicity of Candida species is associated with its ability to form Biofilm and is an essential virulence determinant during candidiasis (Mohandas et al., 2011).
The formation of Candida biofilms carries important clinical repercussions because of their increased resistance to antifungal therapy and the ability of cells within biofilms to withstand host immune defenses. Also, biofilm formation on medical devices can negatively impact the host by causing the failure of the device and by serving as a reservoir or source for future continuing infections. The net effect is that Candida biofilms adversely impact the health of these patients with increasing frequency and severity and with soaring economic sequel. Hence detection of biofilms becomes necessary (Bansal et al., 2016).

In conclusion, the present study shows the predominance of NAC species over $C$. albicans. This helps us understand that the pathogenicity and susceptibility to NAC has increased over the years. Hence, this knowledge is very important in the early initiation of anti-fungal therapy especially in patients suffering from systemic candidiasis such as blood stream infections which have a high mortality. The present study also highlights the fact that HiCrome agar can be cost-effective alternative to the other conventional methods which are time 
consuming. However, the major drawback of this medium is the subjective nature of the identification and inability to differentiate few species. With further modifications in the agar, the use of HiCrome agar may obviate the need for conventional identification methods in routine laboratories.

\section{Acknowledgement}

First of all, I would like to thank Dr. M G Shivaramu our principal for providing me the opportunity to take up research and always keeping me motivated. I would also like to thank Dr. D Venkatesha, Professor and Head of Department of Microbiology, AIMS for his constant support and help with the subject. I am very grateful to my guide Dr. Shwetha D C Assistant Professor, Department of Microbiology, AIMS for her valuable guidance without which I could not have completed the research.

\section{References}

Aydemir O, Demiray T, Köroğlu M, Aydemir Y, Altındiş M 2017. Emerge of nonalbicans Candida species; evaluation of Candida species and antifungal susceptibilities according to years. Biomedical Research; 28(6):2820-2825.

Vignesh Kanna, B, G Amar Kumar, M Swapna, Easow JM 2017. Isolation and identification of Candida species from various clinical samples in a tertiary care hospital. International Journal of Research in Medical Sciences; 5(8):3520-22.

Cavalheiro M, Teixeira MC 2018. Candida Biofilms: Threats, Challenges and Promising Strategies. Front Med(Lausanne);5:28.

Chandak RJ, Mishra B, Thakur A, Loomba PS, Dogra V 2018. Comparison of methods of biofilm detection in urinary Candida isolates and Evaluating its role in Persistent Candiduria. National Journal of Laboratory Medicine; 7(2):MO06-11.

Das K H, Getso M 2016. Distribution of Candida albicans and non-albicans Candida in clinical samples and their intrinsic biofilm production status. International Journal of Medical Science and Public Health; 5(12):244347.

De Oliveira Santos GC, Vasconcelos CC, Lopes AJO, de Sousa Cartagenes MDS, Filho A, do Nascimento FRF, et al., 2018. Candida infections and therapeutic strategies: mechanisms of action for traditional and alternative agents. Front Microbiol; 9:1351. doi:10.3389/fmicb.01351.

Deorukhkar SC, Saini S, Mathew S 2014. Non-albicans Candida infection: An Emerging Threat. Interdisciplinary Perspectives on Infectious Diseases; Available from: http://dx.doi.org/10.1155/2014/615958. Accessed on Dec.7th, 2018.

Dhale RP, Ghorpade MV, Dharmadhikar CA 2014. Comparison of various methods used to detect Biofilm Production of Candida Species. Journal of Clinical and Diagnostic Research;8(11):18-20.

Forbes BA, Sahm DF, Weiss field AS 2007. Laboratory methods in basic Mycology. Bailey and Scott's diagnostic Microbiology $12^{\text {th }}$ edition, USA: Mosby Elsevier; 629-716.

Horvath LL, Hospenthal DR, Murray CK, Dooley DP 2003. Direct isolation of Candida species from blood cultures on the chromogenic medium CHROM agar Candida. Journal of Clinical Microbiology; 41: 2629-32.

Jangla S M, Naidu R, Patel S C 2018. Speciation and antifungal susceptibility testing of Candida isolates in various clinical samples in a tertiary care hospital in Mumbai. International 
Journal of Biomedical Research; 9(3):106-11.

Kaup S, Sankarankutty J, Balasubrahmanya HV, Kulkarni S, Nirmala M 2016. Speciation of Candida using HiCrome Candida Differential Agar. International Journal of Current Microbiology and Applies Sciences; 5(7):267-74.

Kaur R, Dhakad MS, Goyal R, Kumar R 2016. Emergence of non-albicans Candida species and antifungal resistance in intensive care unit patients. Asian Pacific Journal of Tropical Biomedicine; 6(5):455-60.

M Bansal, SA Samant, S Singh, Talukdar A 2016. Phenotypic Detection of Biofilms in Candida Species Isolated from Various Clinical Samples. International Journal of Current Microbiology and Applied Sciences; 5(3):47-56.

Manjunath V, GS Vidya, Sharma A, Prakash MR, Murugesh 2012. Speciation of Candida by HiCrome agar and Sugar assimilation test in both HIV infected and non-infected patients. International Journal of Biological \& Medical Research; 3(2):1778-82.

Marak MB, Dhanashree B 2018. Antifungal Susceptibility and Biofilm Production of Candida Spp. Isolated from Clinical Samples. International Journal of Microbiology: 7495218.

Naveen Saxena, Deepak Maheshwari, Divya Dadhich, Savita Singh 2014. "Evaluation of Congo Red Agar for Detection of Biofilm Production by Various Clinical Candida Isolates". Journal of Evolution of Medical and
Dental Sciences; 3(59):13234-38

Rachana Mehta, Anupama S. Wyawahare 2016. Evaluation of Hicrome Candida Differential agar for Species Identification of Candida Isolates from Various Clinical Samples. International Journal of Contemporary Medical Research;3(4):1219-22.

Sandhu R, Dahiya S, Sharma R K 2015. Isolation and Identification of Candida and Non candida Candida species using chromogenic medium. International Journal of Biomedical Research;6(12):958-62.

Sharma P, Sambhyal SS, Shrivastava D 2017. Phenotypic detection of biofilms in candid species isolated from various clinical specimen. International Journal Of Advanced Research 2017;5(1): 23.

Sardi JCO, Scorzoni L, Bernardi T, et al., 2013. Candida species: current epidemiology, pathogenicity, Biofilm formation, natural antifungal products and new therapeutic options. Journal of Medical Microbiology; 62: 10-24.

Shettar SK, Patil AB, Nadagir SD, Shepur TA, Mythri BA, Gadadavar S 2012. Evaluation of HiCrome differential agar for speciation of Candida. Journal of Academy of Medical Sciences; 2(3):102-4.

V Mohandas and M Ballal 2011. Distribution of Candida species in different clinical samples and their virulence: biofilm formation, proteinase and phospholipase production: a study on hospitalized patients in Southern India. Journal of Global Infectious Diseases; 3(1): 10-14.

\section{How to cite this article:}

Prateeksha, S., D.C. Shwetha and Venkatesha, D. 2020. Isolation and Identification of Candida Species from Various Clinical Samples and their Biofilm Production Status. Int.J.Curr.Microbiol.App.Sci. 9(06): 3630-3638. doi: https://doi.org/10.20546/ijcmas.2020.906.427 